\title{
DUAL ALGORITHMS FOR CONVEX APPROXIMATIONS OF HISTOGRAMS USING CUBIC $C^{1}$-SPLINES
}

\author{
JOCHEN W. SCHMIDT \\ Institute of Numerical Analysis, Technical University of Dresden \\ Mommsenstr. 13, D-01062 Dresden, Germany
}

1. Introduction. On the mesh $\Delta=\left\{x_{0}<x_{1}<\ldots<x_{n}\right\}$ defined on the interval $\left[x_{0}, x_{n}\right]$ let a histogram $F=\left\{f_{1}, \ldots, f_{n}\right\}$ be given, i.e. $f_{i}$ is the frequency for the subinterval $\left[x_{i-1}, x_{i}\right], i=1(1) n$. The local mesh spacing is denoted by $h_{i}=x_{i}-x_{i-1}$. In many practical applications, it is of interest to have a $C^{1}$-function $s$ that satisfies exactly or approximatively the area matching condition

$$
\int_{x_{i-1}}^{x_{i}} s(x) d x=h_{i} f_{i}, \quad i=1(1) n,
$$

and that, in addition, preserves the shape of the histogram $F$. In this paper we are concerned with area matching as well as with smoothing of histograms under convexity constraints. The functions $s$ are assumed to be cubic $C^{1}$-splines on $\Delta$.

The problem of convex area matching has, if it is solvable at all, an infinite number of solutions. Thus it is convenient to have a choice function. In the present paper,

$$
\operatorname{minimize} \int_{x_{0}}^{x_{n}} s^{\prime}(x)^{2} d x
$$

is taken. In smoothing histograms, following [S92] the objective function

$$
\operatorname{minimize} l \int_{x_{0}}^{x_{n}} s^{\prime}(x)^{2} d x+\sum_{i=1}^{n} p_{i}\left(f_{i}-\frac{1}{h_{i}} \int_{x_{i-1}}^{x_{i}} s(x) d x\right)^{2}
$$

1991 Mathematics Subject Classification: 65D07, 41A15.

The paper is in final form and no version of it will be published elsewhere. 
is used. Here $l>0$ is a global parameter while $p_{1}>0, \ldots, p_{n}>0$ are local parameters. The function (1.3) may be considered as an extension of Schoenberg's functional [Sh64] well-known from smoothing data. For another extension see [S91] where the second derivative is used instead of $s^{\prime}$.

There are several models that lead to the constrained approximation of histograms. Here we mention the following one occurring in the one-dimensional motion of a material point: Determine the velocity $s=s(x)$ as a function of the time $x$ such that the point reaches the positions $g_{i}$ at times $x_{i}, i=0(1) n$. In this case, defining the histogram $F$ we have to set

$$
f_{i}=\frac{g_{i}-g_{i-1}}{h_{i}}, \quad i=1(1) n
$$

The derivative $s^{\prime}$ means the acceleration of the material point. Hence, the functional (1.3) can be considered as a trade-off between minimizing the acceleration and the deviation from area matching. Let us remark that in this model constraints like positivity are obvious.

Recently, several papers have appeared concerned with area matching under constraints. We refer to [N82], [SU88], [MC89], [SHN90], [S91], [SH91], [S93] and to the book [Sp90]. In these references various types of polynomial splines are considered. Constrained smoothing of histograms is treated in [S92] using quadratic splines. In the present paper we apply cubic $C^{1}$-splines.

2. Results on dualizing partially separable programs. The problems under consideration are shown to lead to a partially separable program of the type

$$
\begin{array}{ll}
\text { minimize } & \sum_{i=1}^{n} F_{i}\left(y_{i-1}, m_{i-1}, y_{i}, m_{i}\right), \\
\text { subject to } & \left(y_{i-1}, m_{i-1}, y_{i}, m_{i}\right)^{\mathrm{T}} \in W_{i}, \quad i=1(1) n .
\end{array}
$$

Programs of this structure can be solved effectively by dualization; see e.g. [DS85], [SS90], [S92a]. With the Fenchel conjugates $H_{i}^{*}$ to $F_{i}$ and $W_{i}$ defined by

(2.2) $H_{i}^{*}(\varrho, \xi, \sigma, \eta)=\sup \left\{\varrho f+\xi x+\sigma g+\eta y-F_{i}(f, x, g, y):(f, x, g, y)^{\mathrm{T}} \in W_{i}\right\}$

a program dual to $(2.1)$ reads

$$
\begin{aligned}
& \operatorname{maximize} \quad-\sum_{i=1}^{n} H_{i}^{*}\left(y_{i-1}^{*}, m_{i-1}^{*},-y_{i}^{*},-m_{i}^{*}\right) \\
& \text { with } y_{0}^{*}=m_{0}^{*}=y_{n}^{*}=m_{n}^{*}=0 .
\end{aligned}
$$

In the present cases of convexity constraints, the conjugates can be explicitly computed, and they are everywhere finite. Thus, the dual program (2.3) becomes unconstrained. Therefore, from the numerical point of view, dualization is here very effective. Further, the maximizers in the four-dimensional programs (2.2) turn out to be unique. This implies the validity of the return-formula

$$
\left(y_{i-1}, m_{i-1}, y_{i}, m_{i}\right)^{\mathrm{T}}=\operatorname{grad} H_{i}^{*}\left(y_{i-1}^{*}, m_{i-1}^{*},-y_{i}^{*},-m_{i}^{*}\right), \quad i=1(1) n,
$$


by means of which the solution of the primal program can be directly computed if a solution of the dual is known.

3. Cubic $C^{1}$-splines in constrained histogram approximation. A cubic spline $s$ on $\Delta$ can be defined for $x \in\left[x_{i-1}, x_{i}\right]$ by

$$
s(x)=y_{i-1} u+y_{i} t+\left\{\left(y_{i}-y_{i-1}-h_{i} m_{i}\right) t+\left(h_{i} m_{i-1}-y_{i}+y_{i-1}\right) u\right\} t u
$$

when $i=1(1) n$. Here $t$ and $u$ are local variables given by $x=x_{i-1}+t h_{i}$ and $u=1-t$. It follows that $s \in C^{1}\left[x_{0}, x_{n}\right]$ for all parameters $y_{0}, m_{0}, \ldots, y_{n}, m_{n}$, and

$$
s\left(x_{i}\right)=y_{i}, \quad s^{\prime}\left(x_{i}\right)=m_{i}, \quad i=0(1) n .
$$

Further, $s$ is easily proved to be convex on $\left[x_{0}, x_{n}\right]$ if and only if

$$
2 m_{i-1}+m_{i} \leq \frac{3}{h_{i}}\left(y_{i}-y_{i-1}\right) \leq m_{i-1}+2 m_{i}, \quad i=1(1) n ;
$$

see [N78]. The characterization of monotonicity from paper [FC80] reads

$$
\begin{array}{r}
m_{i-1} \geq 0, \quad m_{i} \geq 0, \quad m_{i-1}-\sqrt{m_{i-1} m_{i}}+m_{i} \leq \frac{3}{h_{i}}\left(y_{i}-y_{i-1}\right), \\
i=1(1) n ;
\end{array}
$$

for a positivity criterion we refer to [SH88]. Finally, we get in a straightforward way

$$
\int_{x_{i-1}}^{x_{i}} s(x) d x=\frac{h_{i}}{2}\left(y_{i-1}+y_{i}\right)+\frac{h_{i}^{2}}{12}\left(m_{i-1}-m_{i}\right)
$$

and

$$
\begin{aligned}
\int_{x_{i-1}}^{x_{i}} s^{\prime}(x)^{2} d x= & \frac{h_{i}}{15}\left\{2 m_{i-1}^{2}-m_{i-1} m_{i}+2 m_{i}^{2}\right. \\
& \left.-\frac{3}{h_{i}}\left(y_{i}-y_{i-1}\right)\left(m_{i-1}+m_{i}\right)+\frac{18}{h_{i}^{2}}\left(y_{i}-y_{i-1}\right)^{2}\right\} .
\end{aligned}
$$

3.1. Convex area matching. In view of (3.3), (3.5), there are convex area matching cubic $C^{1}$-splines if and only if the linear system of equalities and inequalities

$$
\begin{aligned}
& 2 m_{i-1}+m_{i} \leq \frac{3}{h_{i}}\left(y_{i}-y_{i-1}\right) \leq m_{i-1}+2 m_{i}, \\
& \frac{1}{2}\left(y_{i-1}+y_{i}\right)+\frac{h_{i}}{12}\left(m_{i-1}-m_{i}\right)=f_{i}, \quad i=1(1) n,
\end{aligned}
$$

is solvable, and every solution $\left(y_{0}, m_{0}, \ldots, y_{n}, m_{n}\right)$ leads via (3.1) to a spline with the desired properties. A solvability criterion for problem (3.7) based on the so-called staircase algorithm [CM84], [SH84] is given in the paper [S91]. This existence test may fail, even when the histogram $F$ is in convex position in the sense of [SHN90]. If the criterion is satisfied then, in general, there exist an infinite 
number of convex histosplines. For selecting one of them the choice function (1.2) is proposed. Thus, because of (3.6) and (3.7) we are led to an optimization problem (2.1) with

$$
F_{i}(f, x, g, y)=\frac{h_{i}}{15}\left\{2 x^{2}-x y+2 y^{2}-\frac{3}{h_{i}}(g-f)(x+y)+\frac{18}{h_{i}^{2}}(g-f)^{2}\right\}
$$

and

$$
\begin{aligned}
W_{i}=\left\{(f, x, g, y)^{\mathrm{T}}:\right. & \\
& \left.\quad 2 x+y \leq \frac{3}{h_{i}}(g-f) \leq x+2 y, \frac{1}{2}(f+g)+\frac{h_{i}}{12}(x-y)=f_{i}\right\} .
\end{aligned}
$$

The corresponding Fenchel conjugates needed for dualizing the program (2.1), (3.8), (3.9) are given in Section 4 of this paper.

3.2. Monotone area matching. When considering monotone histopolation, in the test system (3.7) we have to replace (3.3) by the monotonicity condition (3.4). If now $m_{0}=\ldots=m_{n}=0$ is set, the system

$$
y_{i-1} \leq y_{i}, \quad \frac{1}{2}\left(y_{i-1}+y_{i}\right)=f_{i}, \quad i=1(1) n,
$$

arises which characterizes $F$ to be in monotone position; see [SHN90]. Thus, there exist monotone area matching cubic $C^{1}$-splines if the histogram is in monotone position. The number of these splines is in general infinite. Again the choice function (1.2) is proposed leading to a program (2.1) with $F_{i}$ defined by (3.8) and

$$
\begin{aligned}
W_{i}=\left\{(f, x, g, y)^{\mathrm{T}}\right. & : x \geq 0, y \geq 0, \\
& \left.x-\sqrt{x y}+y \leq \frac{3}{h_{i}}(g-f), \frac{1}{2}(f+g)+\frac{h_{i}}{12}(x-y)=f_{i}\right\} .
\end{aligned}
$$

Here it seems to be difficult to compute the Fenchel conjugates explicitly. Thus, this must be done numerically.

3.3. Convex smoothing of histograms. Using the objective function (1.3) this problem can be formulated by a program (2.1) with

$$
\begin{aligned}
F_{i}(f, x, g, y)= & \frac{l h_{i}}{15}\left\{2 x^{2}-x y+2 y^{2}-\frac{3}{h_{i}}(g-f)(x+y)+\frac{18}{h_{i}^{2}}(g-f)^{2}\right\} \\
& +p_{i}\left\{f_{i}-\frac{1}{2}(f+g)-\frac{h_{i}}{12}(x-y)\right\}^{2}
\end{aligned}
$$

and

$$
W_{i}=\left\{(f, x, g, y)^{\mathrm{T}}: 2 x+y \leq \frac{3}{h_{i}}(g-f) \leq x+2 y\right\} .
$$

Now, the feasible domain is non-empty. E.g., the point with $y_{0}=m_{0}=\ldots=$ $y_{n}=m_{n}=0$ satisfies the constraints. Further, the Hessian of the objective 
function is positive-definite. Thus, the program (2.1), (3.12), (3.13) is always uniquely solvable. The solution can be determined via dualization (2.3). The Fenchel conjugates needed there are computed in an explicit manner in Section 4.

4. Determination of Fenchel conjugates. As pointed out above, the conjugates (2.2) are essential in the dual (2.3) as well as in the return-formula (2.4).

4.1. Conjugates in convex area matching. In the case (3.8), (3.9) of convex area matching, the conjugates (2.2) read

$$
H_{i}^{*}(\varrho, \xi, \sigma, \eta)=K_{i}^{*}(\varrho, \xi, \sigma, \eta ; 1)+L_{i}^{*}(\varrho, \xi, \sigma, \eta ; 1),
$$

where

$$
\begin{aligned}
K_{i}^{*}(\varrho, \xi, \sigma, \eta ; l)= & f_{i}(\varrho+\sigma)+\frac{h_{i}}{12 l}\left(\varrho^{2}-\varrho \sigma+\sigma^{2}\right) \\
& +\frac{1}{2 l}(\sigma \eta-\varrho \xi)+\frac{3}{4 h_{i} l}\left(3 \xi^{2}+2 \xi \eta+3 \eta^{2}\right)
\end{aligned}
$$

and

$$
\begin{aligned}
& L_{i}^{*}(\varrho, \xi, \sigma, \eta ; l) \\
& \qquad \begin{array}{l}
0 \quad \text { for } \varrho+\sigma \geq 12(3 \xi+2 \eta) / h_{i}, \varrho+\sigma \geq-12(2 \xi+3 \eta) / h_{i} \\
-h_{i}\left\{-(\varrho+\sigma) / 4+3(3 \xi+2 \eta) / h_{i}\right\}^{2} /(48 l) \\
\quad \text { for } \varrho+\sigma \leq 12(3 \xi+2 \eta) / h_{i}, \varrho+\sigma \geq 4(\xi-2 \eta) / h_{i} \\
-h_{i}\left\{-(\varrho+\sigma) / 4-3(2 \xi+3 \eta) / h_{i}\right\}^{2} /(48 l) \\
\quad \text { for } \varrho+\sigma \leq-12(2 \xi+3 \eta) / h_{i}, \varrho+\sigma \geq 4(2 \xi-\eta) / h_{i} \\
-h_{i}(\varrho+\sigma)^{2} /(48 l)+1(\varrho+\sigma)(\xi-\eta) /(4 l)-1\left(2 \xi^{2}+\xi \eta+2 \eta^{2}\right) /\left(h_{i} l\right) \\
\quad \text { for } \varrho+\sigma \leq 4(\xi-2 \eta) / h_{i}, \varrho+\sigma \leq 4(2 \xi-\eta) / h_{i} .
\end{array}
\end{aligned}
$$

Proof of formula (4.1). With the Lagrangian $\Phi$ for program (2.2), (3.8), (3.9) given by

$$
\begin{aligned}
\Phi(f, x, g, y, \alpha, \lambda, \mu)=-\varrho f-\xi x-\sigma g-\eta y \\
+\frac{h_{i} l}{15}\left\{2 x^{2}-x y+2 y^{2}-\frac{3}{h_{i}}(g-f)(x+y)+\frac{18}{h_{i}^{2}}(g-f)^{2}\right\} \\
+\alpha\left(\frac{g+f}{2}+\frac{h_{i}}{12}(x-y)-f_{i}\right)+\lambda\left(2 x+y-\frac{3}{h_{i}}(g-f)\right) \\
+\mu\left(\frac{3}{h_{i}}(g-f)-x-2 y\right),
\end{aligned}
$$

where $l=1$, the Kuhn-Tucker conditions read as follows:

$$
\begin{aligned}
& \Phi_{x}=-\xi+\frac{h_{i} l}{15}\left\{4 x-y-\frac{3}{h_{i}}(g-f)\right\}+\frac{\alpha h_{i}}{12}+2 \lambda-\mu=0, \\
& \Phi_{y}=-\eta+\frac{h_{i} l}{15}\left\{4 y-x-\frac{3}{h_{i}}(g-f)\right\}-\frac{\alpha h_{i}}{12}+\lambda-2 \mu=0,
\end{aligned}
$$




$$
\begin{aligned}
& \Phi_{f}=-\varrho+\frac{h_{i} l}{15}\left\{\frac{3}{h_{i}}(x+y)-\frac{36}{h_{i}^{2}}(g-f)\right\}+\frac{\alpha}{2}+\frac{3}{h_{i}}(\lambda-\mu)=0, \\
& \Phi_{g}=-\sigma+\frac{h_{i} l}{15}\left\{-\frac{3}{h_{i}}(x+y)+\frac{36}{h_{i}^{2}}(g-f)\right\}+\frac{\alpha}{2}-\frac{3}{h_{i}}(\lambda-\mu)=0, \\
& \Phi_{\alpha}=0, \quad \Phi_{\lambda} \leq 0, \quad \lambda \geq 0, \quad \lambda \Phi_{\lambda}=0, \quad \Phi_{\mu} \leq 0, \quad \mu \geq 0, \quad \mu \Phi_{\mu}=0 .
\end{aligned}
$$

By means of (4.5), (4.6) and (4.7), (4.8) we get

$$
\begin{array}{r}
-\xi-\eta+\frac{h_{i} l}{5}\left\{x+y-\frac{2}{h_{i}}(g-f)\right\}+3(\lambda-\mu)=0, \\
-\varrho+\sigma+\frac{l}{5}\left\{2(x+y)-\frac{24}{h_{i}}(g-f)\right\}-\frac{6}{h_{i}}(\lambda-\mu)=0
\end{array}
$$

as well as

$$
\begin{aligned}
-\xi+\eta+\frac{h_{i} l}{3}(x-y)+\frac{\alpha h_{i}}{6}+\lambda+\mu & =0, \\
-\varrho-\sigma+\alpha & =0 .
\end{aligned}
$$

Now, from (4.10), (4.11) and (4.12), (4.13) we compute

$$
\begin{aligned}
& x+y=\frac{\sigma-\varrho}{2 l}+\frac{6}{h_{i} l}(\xi+\eta)-\frac{15}{h_{i} l}(\lambda-\mu), \\
& x-y=-\frac{\varrho+\sigma}{2 l}+\frac{3}{h_{i} l}(\xi-\eta)-\frac{3}{h_{i} l}(\lambda+\mu),
\end{aligned}
$$

implying

$$
\begin{aligned}
& x=-\frac{\varrho}{2 l}+\frac{3}{2 h_{i} l}(3 \xi+\eta)-\frac{3}{h_{i} l}(3 \lambda-2 \mu), \\
& y=\frac{\sigma}{2 l}+\frac{3}{2 h_{i} l}(\xi+3 \eta)-\frac{3}{h_{i} l}(2 \lambda-3 \mu) .
\end{aligned}
$$

Further, from (4.10), (4.11) and from $\Phi_{\alpha}=0$ we obtain

$$
\begin{aligned}
& g-f=\frac{h_{i}}{4 l}(\sigma-\varrho)+\frac{\xi+\eta}{2 l}, \\
& g+f=2 f_{i}+\frac{h_{i}}{12 l}(\varrho+\sigma)+\frac{\eta-\xi}{2 l}+\frac{\lambda+\mu}{2 l} .
\end{aligned}
$$

Hence it follows that

$$
\begin{aligned}
& f=f_{i}+\frac{h_{i}}{12 l}(2 \varrho-\sigma)-\frac{\xi}{2 l}+\frac{\lambda+\mu}{4 l}, \\
& g=f_{i}+\frac{h_{i}}{12 l}(2 \sigma-\varrho)+\frac{\eta}{2 l}+\frac{\lambda+\mu}{4 l} .
\end{aligned}
$$

In view of the complementarity conditions (4.9) there are four different cases to be discussed. 
Case 1: Let $\lambda=0, \mu=0$. Then $\Phi_{\lambda} \leq 0, \Phi_{\mu} \leq 0$ lead to the domain of validity

$$
\varrho+\sigma \geq \frac{12}{h_{i}}(3 \xi+2 \eta), \quad \varrho+\sigma \geq-\frac{12}{h_{i}}(2 \xi+3 \eta) .
$$

Further, the maximizer resulting from (4.16), (4.17), (4.20), (4.21),

$$
\begin{aligned}
& f=f_{i}+\frac{h_{i}}{12 l}(2 \varrho-\sigma)-\frac{\xi}{2 l}, \\
& x=-\frac{\varrho}{2 l}+\frac{3}{2 h_{i} l}(3 \xi+\eta), \\
& g=f_{i}+\frac{h_{i}}{12 l}(2 \sigma-\varrho)+\frac{\eta}{2 l}, \\
& y=\frac{\sigma}{2 l}+\frac{3}{2 h_{i} l}(\xi+3 \eta)
\end{aligned}
$$

turns out to be unique. Next, using the property that the maximizer satisfies

$$
(f, x, g, y)^{\mathrm{T}}=\operatorname{grad} H_{i}^{*}(\varrho, \xi, \sigma, \eta),
$$

it follows straightforwardly that $H_{i}^{*}(\varrho, \xi, \sigma, \eta)=K_{i}^{*}(\varrho, \xi, \sigma, \eta ; 1)$. Thus, in Case 1 formula (4.1) is proven.

Case 2: Let $\Phi_{\lambda}=0, \mu=0$. These assumptions imply

$$
\lambda=\frac{h_{i}}{24}\left\{-\frac{\varrho+\sigma}{4}+\frac{3}{h_{i}}(3 \xi+2 \eta)\right\},
$$

and $\lambda \geq 0, \Phi_{\mu} \leq 0$ give the domain of validity

$$
\varrho+\sigma \leq \frac{12}{h_{i}}(3 \xi+2 \eta), \quad \varrho+\sigma \geq \frac{4}{h_{i}}(\xi-2 \eta) .
$$

The components of the unique maximizer are obtained by adding the terms

$$
\frac{\lambda}{4 l}, \quad-\frac{9 \lambda}{h_{i} l}, \quad \frac{\lambda}{4 l}, \quad-\frac{6 \lambda}{h_{i} l}
$$

to the values (4.22), respectively. Hence, because of (4.23), we have to add the present term $L_{i}^{*}(\varrho, \xi, \sigma, \eta ; 1)$ to $K_{i}^{*}(\varrho, \xi, \sigma, \eta ; 1)$ in order to get $H_{i}^{*}(\varrho, \xi, \sigma, \eta)$.

Case 3: Let $\lambda=0, \Phi_{\mu}=0$. This case is treated analogously to Case 2.

Case 4: Let $\Phi_{\lambda}=0, \Phi_{\mu}=0$. Now, using (4.16), (4.17), (4.20), (4.21) we find

$$
\lambda=\frac{h_{i}}{12}\left\{-\varrho-\sigma+\frac{4}{h_{i}}(2 \xi-\eta)\right\}, \quad \mu=\frac{h_{i}}{12}\left\{-\varrho-\sigma+\frac{4}{h_{i}}(\xi-2 \eta)\right\},
$$

and $\lambda \geq 0, \mu \geq 0$ describes the domain of validity. The components of the unique maximizer are obtained by adding

$$
\begin{array}{ll}
\frac{\lambda+\mu}{4 l}=-\frac{h_{i}}{24 l}(\varrho+\sigma)+\frac{\xi-\eta}{4 l}, & -\frac{3}{h_{i} l}(3 \lambda-2 \mu)=\frac{\varrho+\sigma}{4 l}-\frac{4 \xi+\eta}{h_{i} l}, \\
\frac{\lambda+\mu}{4 l}=-\frac{h_{i}}{24 l}(\varrho+\sigma)+\frac{\xi-\eta}{4 l}, & -\frac{3}{h_{i} l}(2 \lambda-3 \mu)=-\frac{\varrho+\sigma}{4 l}-\frac{\xi+4 \eta}{h_{i} l}
\end{array}
$$


to the components of (4.22). Property (4.23) again shows that $H_{i}^{*}(\varrho, \xi, \sigma, \eta)$ is obtained if the present $L_{i}^{*}(\varrho, \xi, \sigma, \eta ; 1)$ is added to $K_{i}^{*}(\varrho, \xi, \sigma, \eta ; 1)$.

Thus, the proof of formula (4.1) is complete.

4.2. Conjugates in convex smoothing of histograms. In the case (3.12), (3.13) of convex smoothing of histograms, the conjugates are

$$
H_{i}^{*}(\varrho, \xi, \sigma, \eta)=K_{i}^{*}(\varrho, \xi, \sigma, \eta ; l)+L_{i}^{*}(\varrho, \xi, \sigma, \eta ; l)+\frac{(\varrho+\sigma)^{2}}{4 p_{i}},
$$

where $K_{i}^{*}$ and $L_{i}^{*}$ are defined by (4.2) and (4.3).

Pro of of for mula (4.24). We follow the lines of 4.1. The basic expressions (4.16), (4.17), (4.20), (4.21) for the maximizer remain valid, except that we have to add the term $(\varrho+\sigma) /\left(2 p_{i}\right)$ in (4.20) as well as in (4.21). In view of (4.23) this modification implies the additional term $(\varrho+\sigma)^{2} /\left(4 p_{i}\right)$ in (4.24) relative to (4.1).

5. Computational comments. The described dualization technique leads to the following general approach for solving partially separable programs. ically.

S te p 1: Compute a solution of the unconstrained dual program (2.3) numer-

S tep 2: Determine the solution of program (2.1) by means of the explicit return-formula (2.4).

In Step 1 computer tests with various types of partially separable programs have shown that Newton's method combined with the steepest descent method for supplying sufficiently good starting vectors is effective. In both methods the special structure of the dual (2.3) can be taken into account. Numerical tests in convex smoothing of histograms are done by our students C. Gemmer and G. Prokert. Though the rough initial vector having only zero components is used, the number $N S$ of Newton steps is moderate. The splines in Figure 1 result by setting

\begin{tabular}{l|cc|c}
\hline & $l$ & $p_{i}$ & $N S$ \\
\hline spline 1 & 10 & 1 & 4 \\
spline 2 & 0.1 & 1 & 6 \\
spline 3 & 0.0001 & 1 & 8 \\
\hline
\end{tabular}

while the splines in Figure 2 are obtained for

\begin{tabular}{l|cc|c}
\hline & $l$ & $p_{i}$ & $N S$ \\
\hline spline 1 & 10 & 1 & 8 \\
spline 2 & 0.1 & 1 & 7 \\
spline 3 & 0.0001 & 1 & 18 \\
\hline
\end{tabular}

In Figure 3 we have taken

\begin{tabular}{l|cc|c}
\hline & $l$ & $p_{i}$ & $N S$ \\
\hline spline 1 & 10 & 1 & 10 \\
spline 2 & 0.1 & 1 & 12 \\
\hline
\end{tabular}




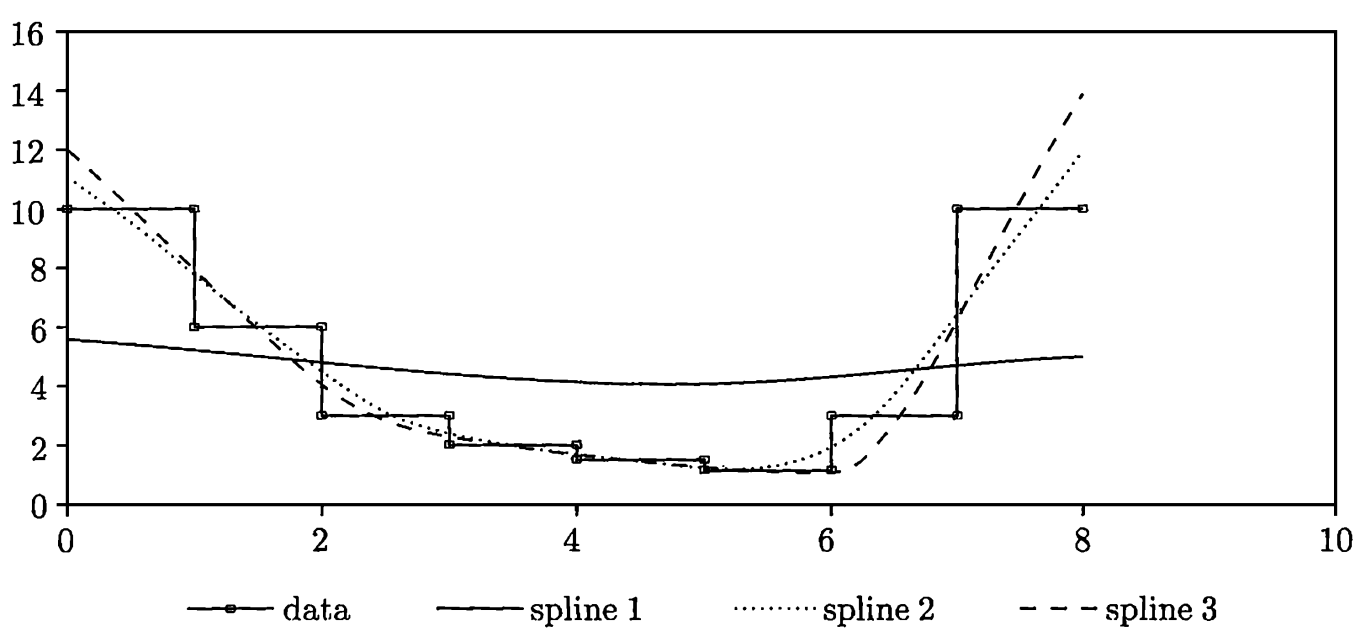

Fig. 1

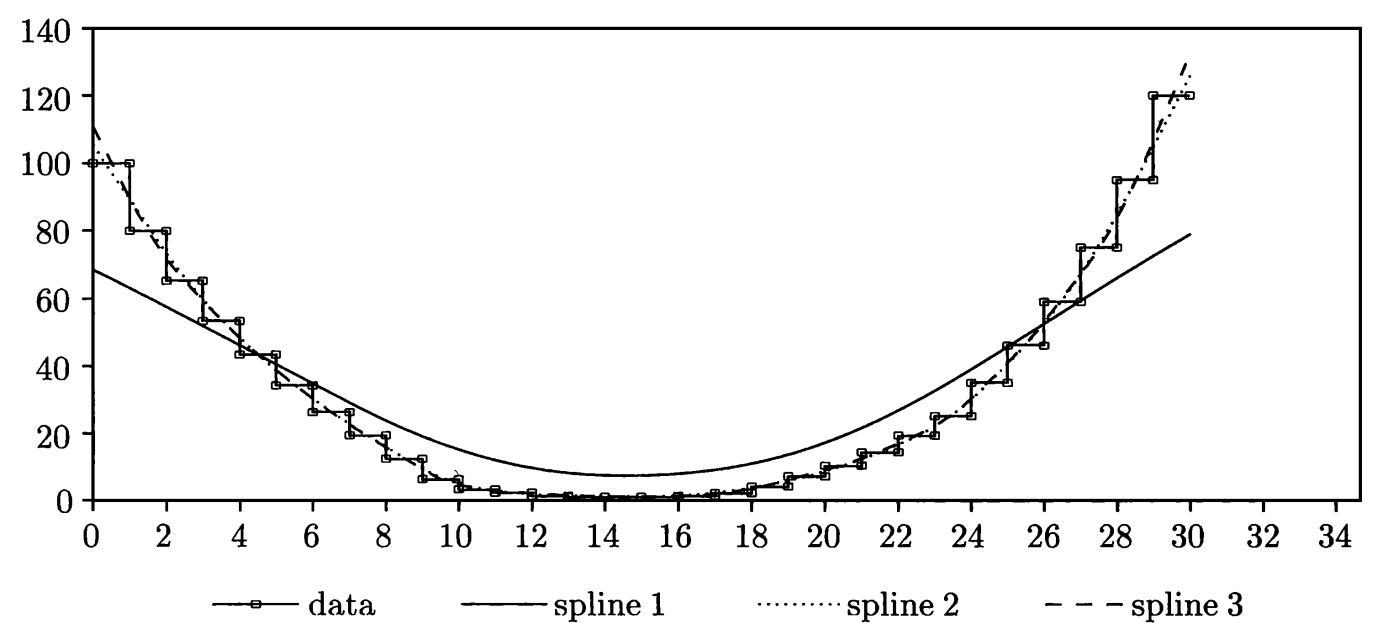

Fig. 2

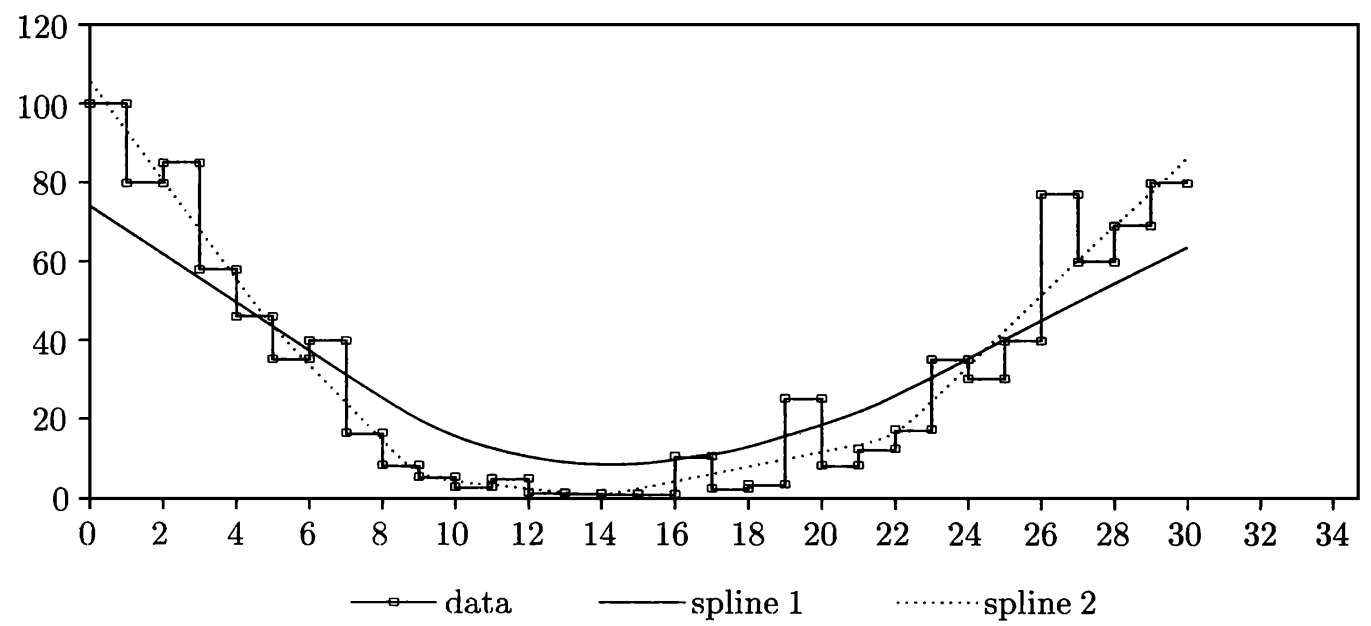

Fig. 3 
Finally, let us remark that in general, for instance in the case of monotone histogram approximation, also the conjugates must be computed numerically. It is of importance that this can be done simultaneously by solving a possibly larger number of four-dimensional programs (2.2).

\section{References}

[CM84] P. Costantini and R. Morandi, Monotone and convex cubic spline interpolation, Calcolo 21 (1984), 281-294.

[DS85] S. Dietze and J. W. Schmidt, Determination of shape preserving spline interpolants with minimal curvature via dual programs, J. Approx. Theory 52 (1988), 43-57.

[FC80] F. N. Fritsch and R. E. Carlson, Monotone piecewise cubic interpolation, SIAM J. Numer. Anal. 17 (1980), 238-246.

[MC89] R. Morandi and P. Costantini, Piecewise monotone quadratic histosplines, SIAM J. Sci. Statist. Comput. 10 (1989), 397-406.

[N78] E. Neuman, Uniform approximation by some Hermite interpolating splines, J. Comput. Appl. Math. 4 (1978), 7-9.

[N82] - Shape preserving interpolation by polynomial splines, Wrocław Univ., Inst. of Comput. Sci. Report No. 122 (1982).

[SU88] M. Sakai and R. A. Usmani, A shape preserving area true approximation of histograms by rational splines, BIT 28 (1988), 329-339.

[S91] J. W. Schmidt, Beiträge zur konvexen Interpolation, Histopolation und Approximation durch Spline-Funktionen, Mitt. Math. Gesellsch. Hamburg 12 (1991), 603-628.

[S92] —, Constrained smoothing of histograms by quadratic splines, Computing 48 (1992), 97-107.

[S92a] -, Dual algorithms for solving convex partially separable optimization problems, Jahresber. Deutsch. Math.-Verein. 94 (1992) 40-62.

[S93] - Positive, monotone, and S-convex $C^{1}$-histopolation on rectangular grids, Computing 50 (1993), 19-30.

[SH84] J. W.Schmidt und W. Heß, Schwach verkoppelte Ungleichungssysteme und konvexe Spline-Interpolation, Elem. Math. 39 (1984), 85-95.

[SH88] - - - Positivity of cubic polynomials on intervals and positive spline interpolation, BIT 28 (1988), 340-352.

[SH91] - - - Shape preserving $C^{2}$-spline histopolation, Hamburger Beitr. Angew. Math., preprint A 41 (1991), and J. Approx. Theory, to appear.

[SHN90] J. W. Schmidt, W. Heß and T. Nordheim, Shape preserving histopolation using rational quadratic splines, Computing 44 (1990), 245-258.

[SS90] J. W. Schmidt and I. Scholz, A dual algorithm for convex-concave data smoothing with cubic $C^{2}$-splines, Numer. Math. 57 (1990), 330-350.

[Sh64] I. J. Schoenberg, Spline functions and the problem of graduation, Proc. Nat. Acad. Sci. U.S.A. 52 (1964), 947-950.

[Sp90] H. Späth, Eindimensionale Spline-Interpolations-Algorithmen, R. Oldenbourg-Verlag, München-Wien 1990. 